\title{
Orientação empreendedora e ambidestria organizacional: uma pesquisa com profissionais de TI
}

\section{Entrepreneurial orientation and organizational ambidexterity: a survey with professionals of IT}

\author{
LETÍCIA M. VARGAS* \\ ELVIS SILVEIRA-MARTINS ${ }^{* *}$
}

\section{RESUMO}

Estudos sobre o ato de empreender na atual interdependência global permitem expandir as discussões sobre o processo de gestão associado à orientação empreendedora e ambidestria organizacional. Nesse sentido, o presente trabalho tem por objetivo identificar os traços da orientação empreendedora em associação com a ambidestria organizacional em profissionais da área de Tecnologia da Informação por meio de uma análise sob múltiplos ângulos. A pesquisa foi realizada com base em uma amostra de 153 profissionais da área de TI do Brasil, utilizando metodologia quantitativa e técnica survey. Considerando as hipóteses que foram possíveis suportar, os resultados apontam para uma correlação positiva da dimensão inovação da orientação empreendedora com a ambidestria e suas dimensões.

Palavras-chave: Orientação empreendedora; ambidestria; estratégia.

Universidade Federal de Pelotas. Mestranda em Desenvolvimento Territorial e Sistemas Agroindustriais e Bacharel em Administração pela Universidade Federal de Pelotas. Tecnóloga em Análise e Desenvolvimento de Sistemas pelo Centro Universitário Claretiano, Bacharel em Direito pela Universidade Católica de Pelotas, Especialista em Engenharia de Sistemas pela ESAB. le.mvargas@gmail.com

* Universidade Federal de Pelotas. Professor Permanente do Programa de Pós-Graduação em Desenvolvimento Territorial e Sistemas Agroindustriais e do Mestrado Profissional em Administração Pública. Administrador, Mestre em Administração e Doutor em Administração e Turismo. elvis.professor@gmail.com 


\section{Abstract}

Studies on entrepreneurship in the current global interdependence allow you to expand discussions on the management process associated with entrepreneurial orientation and organizational ambidexterity. In this sense, this paper aims to identify the traits of entrepreneurial orientation in association with organizational ambidexterity in professional Information Technology area through an analysis from multiple angles. The research was carried out based on a sample of 153 professionals in the field of this area in Brazil, using quantitative methodology and technical survey. Considering the hypotheses that were possible support, the results of this study point to a positive correlation dimension of innovation entrepreneurial orientation with ambidexterity and its dimensions.

Keywords: Entrepreneurial orientation; ambidexterity; strategy.

\section{INTRODUÇÃo}

O empreendedorismo é identificado constantemente em pesquisas de diferentes campos científicos, fazendo com que a interdependência global das economias, bem como as mudanças sociais intensas e dinâmicas criem um contexto rico para a compreensão dos elementos que compõem o ato de empreender, além dos gatilhos para o início da atividade empresarial. De acordo com Schumpeter (1982) o empreendedor é o indivíduo que, por meio do desenvolvimento de novos produtos e serviços, destrói a ordem econômica existente, apresentando-se como elemento central para a inovação na sociedade.

Nesse sentido, compreender o empreendedorismo de forma mais ampla, conforme sugere Miller (1983), permite expandir as discussões sobre as constantes mudanças organizacionais que exigem esforços diferenciados dos gestores e tomadores de decisão. Dessa forma, a orientação empreendedora se apresenta como um caminho teórico para a compreensão do processo de gestão associado interconectado com o empreendedorismo.

Assim, quando analisada a propensão individual para empreender, pode-se destacar a importância da identificação dos traços empreendedores na adoção de novas estratégias e tecnologias. Segundo Göktan e Gupta (2013), a orientação empreendedora abarca crenças 
e valores associados com a tendência a ser proativo, a assumir riscos e a inovar, e ao interpretar como estas se relacionam com outros fatores internos e externos, caminhos estratégicos são traçados tanto em nível organizacional quanto individual, podendo ser um diferencial no dinamismo econômico e social atual.

Entretanto, segundo Morris (1998), existe uma dificuldade relacionada à existência de instrumentos para identificar o comportamento empreendedor e suas associações com outros construtos da área estratégica. Nesse contexto, a pesquisa realizada por Bolton e Lane (2012) acrescenta um novo olhar à compreensão de comportamentos empreendedores e escolhas estratégicas empresariais. De acordo com Taatila e Down (2012), existe uma crescente ênfase no desenvolvimento e encorajamento das tendências e das práticas empresariais em todos os níveis organizacionais; assim, estudos sobre a orientação empreendedora individual são oportunos e relevantes.

Observa-se que a necessidade de construção e desconstrução das organizações para sua manutenção no mercado cresceu ao longo das últimas décadas. Desenvolveu-se um binômio entre as necessidades de curto e longo prazo, no qual de um lado existe a obrigação de aumentar a estabilidade estratégica para manutenção das atividades já existentes e, de outro lado, o imperativo da revolução constante para manter competitividade. Essas características são abarcadas pelo conceito de ambidestria que se apresenta como estratégia para direcionamento das ações de desenvolvimento organizacional, como afirmam Tushman e O’Reilly (1996). Dessa maneira, a ambidestria organizacional emerge como uma capacidade organizacional em que as ações de exploração (criação) e explotação (melhoria) competem igualmente pelo protagonismo nas tomadas de decisão.

Nesse sentido, procurou-se identificar um setor/segmento que possuísse as condições para verificar o posicionamento empreendedor, além de apresentar estratégias voltadas para a melhoria e criação de novos produtos/serviços. Segundo Carvalho Junior (2005), o setor de Tecnologia de Informação (TI) se relaciona diretamente com a inovação de novas formas de fazer, influenciando diretamente o desenvolvimento de diferentes áreas da economia. Ademais, é potencializado pelo empreendedorismo em organizações de diferentes tamanhos e espectros. 
Com base nesse cenário, o objetivo da presente pesquisa é verificar a associação entre as dimensões da orientação empreendedora individual e as dimensões da ambidestria organizacional de maneira uni e multidimensional.

Ainda, torna-se importante destacar que o desenvolvimento deste estudo recebe respaldo na carência de outros que procurem associar a orientação empreendedora com a ambidestria. Portanto, além de proporcionar reflexões sobre essa conjectura, a pesquisa ainda amplia a discussão, incluindo a análise sobre diferentes prismas (uni/multidimensionalidade).

Assim, pesquisa é composta por três seções. A primeira é composta pela fundamentação teórica dos construtos orientação empreendedora e ambidestria, além da apresentação das hipóteses de estudo. Na segunda seção, são descritos os procedimentos metodológicos adotados no desenvolvimento da pesquisa, e a quarta abrange a análise dos dados e resultados obtidos.

\section{FUndaMENTAÇão TEÓRICA}

Na presente seção são apresentados os direcionamentos teóricos que conduzem esta pesquisa. Serão abordados os construtos orientação empreendedora e ambidestria, além de apresentar as hipóteses trabalhadas.

\subsection{Orientação empreendedora}

Os estudos relacionados à orientação empreendedora têm origem em trabalho realizado por Miller e Friesen (1982), no qual, baseados na aplicação do conceito de empreendedorismo à organização, desenvolveram um novo olhar sobre o processo de inovação, demonstrando serem as mudanças parte do processo natural de crescimento empresarial.

Em trabalho posterior, Miller (1983) afirma que uma organização possui uma orientação empreendedora se reunir em suas características fundamentais a busca constante pela inovação de seus produtos e serviços, assumindo riscos inerentes ao processo e sendo proativa em relação à concorrência. Em outras palavras, segundo o estudo de Miller (1983), a orientação empreendedora somente está presente em empresas que possuem um equilíbrio do 
trinômio composto por inovação, proatividade e assunção de riscos, considerando, dessa forma, a orientação empreendedora como um construto unidimensional.

De acordo com Stevenson e Jarillo (1990), a orientação empreendedora reflete os métodos e as práticas adotadas como estratégia pela empresa para tomada de decisão, emergindo da noção de oportunidade de novos negócios que podem favorecer o desenvolvimento da organização. Nessa mesma linha, Covin e Slevin (1991) afirmam que a atitude empreendedora é percebida mediante um padrão comportamental que permeia os níveis estratégico, tático e operacional da organização, apresentando-se como filosofia norteadora das atividades empresariais.

Com base nos estudos realizados por Miller (1983), Lumpkin e Dess (1996) expandiram a pesquisa, ampliando a orientação empreendedora para uma estrutura de cinco dimensões: inovação, assunção de riscos, proatividade, autonomia e agressividade competitiva. De acordo com esse estudo, elas estão presentes em maior ou menor medida conforme fatores internos e externos, ou ainda, consoante as características dos líderes da organização, e quando combinados entre si auxiliam na identificação dos melhores caminhos estratégicos a serem adotados pela empresa. Essa ampliação do construto apresenta a multidimensionalidade da orientação empreendedora, posição corroborada por Morris (1998) ao afirmar que o contexto no qual a organização está inserida altera a combinação de dimensões empreendedoras afloradas.

A orientação empreendedora é associada positivamente ao crescimento empresarial, por existir relação direta com a forma de gerenciamento dos processos de uma empresa, tornando-se elemento distintivo em um ambiente de inovação contínua (TEECE, 2007). Nesse sentido, Kollmann, Chistofor e Kuckertz (2007), afirmam que a orientação empreendedora abrange tanto o nível empresarial quanto o individual, e ambos são importantes no desenvolvimento das estratégias organizacionais. Para Dickson e Weaver (2008), a presença dos elementos que compõem o construto no rol de atributos pessoais indica uma propensão ao empreendedorismo, que, em conjunto com características ambientais, influencia a tomada de decisão. 
De acordo com Bolton e Lane (2012), as características individuais influenciam as tomadas de decisão, impactando diretamente a trajetória estratégica da organização. Portanto, a identificação da orientação empreendedora individual permite compreender comportamentos e escolhas particulares em um ambiente de trabalho. A pesquisa realizada por esses autores identificou que, entre as dimensões da orientação empreendedora propostas pela literatura, aquelas sugeridas por Miller (1983) são as que possuem aderência em nível individual, razão por que foram utilizadas na presente pesquisa.

A proatividade corresponde às iniciativas para antecipar e perseguir novas oportunidades, moldando o ambiente com a inserção de novos serviços e produtos (FREITAS et al., 2012). A essência da proatividade está atrelada ao coeficiente de apoio ao desenvolvimento de antecipação e implementação de inovações de maneira a se destacar no mercado. Segundo Faoro e Abreu (2016), uma empresa é proativa não só quando se posiciona a frente de seus concorrentes, mas também quando está na vanguarda no reconhecimento de seus clientes.

A dimensão inovatividade é tida pela literatura como elemento central da orientação empreendedora, envolvendo a criatividade e a experimentação. Segundo Lazzarotti et al. (2015), o comportamento inovador tem suas bases na criatividade, na percepção de lacunas no mercado e na predisposição empreendedora dos indivíduos, possibilitando o desenvolvimento de novas combinações econômicas. Inovar engloba desde a visão de uma nova oportunidade, do planejamento e do desenvolvimento do produto ou serviço até sua inserção no mercado; ou seja, trata-se de um processo iterativo na natureza (FAORO; ABREU, 2016).

Já a dimensão assunção de riscos está relacionada ao grau de engajamento em projetos de risco, além do grau de ousadia na tomada de decisões e estratégias (FREITAS et al., 2012). Segundo Lazzarotti et al. (2015), pode-se considerar consenso na literatura a aceitação do risco como elemento essencial ao empreendedorismo. Essa relação se demonstra conforme o nível de comprometimento de recursos em novos projetos e desenvolvimento de oportunidades.

Segundo Ferreira et al. (2016), as pesquisas sobre a orientação empreendedora individual ainda são incipientes e necessitam de 
maior discussão e apropriação, principalmente pela potencial contribuição desse construto na gestão e identificação de profissionais estratégicos para o crescimento organizacional.

\subsection{Ambidestria}

O conceito de ambidestria teve origem no campo da gestão da inovação, com o modelo construído por Ducan (1974), o qual está dividido em duas dimensões - iniciação de criação e implementação para a criação -, e tem como foco a estrutura e o processo das organizações consideradas inovadoras. A primeira dimensão abarca um grau maior de complexidade e menor formalização de maneira a facilitar a coleta e o processamento das informações. Já a segunda compreende um grau maior de formalização e menor complexidade, reduzindo conflitos organizacionais e possíveis ambiguidades.

Posteriormente, March (1991) ampliou os estudos de Ducan (1974) ao desenvolver a teoria da ambidestria como um equilíbrio entre ações de exploração e explotação. Nesse sentido, as ações de exploração são todas aquelas relacionadas a gestão de riscos, experimentação, flexibilidade, desenvolvimento de novos produtos e serviços. A explotação, por sua vez, reúne ações de aprimoramento, escolha, implementação e realização dos processos da organização (TUSHMAN; O’REILLY, 1996).

Nesse contexto, Smith e Tushman (2005) afirmam que a exploração e a explotação são vistas como contraditórias por muitas empresas, entretanto o objetivo da ambidestria é o desempenho organizacional eficiente e sustentável. Segundo Gupta, Smith e Shalley (2006), deve existir uma sinergia entre as práticas de exploração e explotação de recursos de forma a permitir um aprimoramento de ambos e consequente crescimento da empresa; todavia, não é necessária a simultaneidade das ações para esse desenvolvimento.

Os benefícios da ambidestria são ratificados nas pesquisas de Lin, Yang e Demirkan (2007) e de Han e Celly (2008), as quais afirmam que a adoção da ambidestria como estratégia da empresa auxilia no desenvolvimento dela, independentemente da disponibilidade de recursos e da idade da empresa.

Assim, de acordo com Bledow et al. (2009), ambidestria pode ser definida como a capacidade de desenvolver e utilizar novos recursos 
e competências (explotação) concomitantemente com o uso eficiente dos recursos já existentes (exploração), administrando com sucesso a dicotomia de criação e manutenção de produtos e/ou serviços. Dessa forma, existe a necessidade de constante atualização dos processos organizacionais de maneira a manter o nível competitivo das empresas; nesse caso, cabe ao gestor a definição dos caminhos a percorrer (TAYLOR; HELFAT, 2009).

De acordo com Hsu, Lien e Chen (2013), o investimento em estratégias ambidestras gera maior desempenho organizacional em relação a outras empresas que não investem nessas práticas. Corroborando esse estudo, Silveira-Martins, Rossetto e Añaña (2014) verificaram que as ações de explotação, exploração ou ambidestras têm efeito favorável no desenvolvimento de empresas, e ao realizar a análise em vinícolas brasileiras confirmaram a relação positiva entre investimento e crescimento empresarial.

Segundo Parmentier e Picq (2016), alcançar a ambidestria é uma atividade complexa, pois as dimensões de exploração e explotação se relacionam com tipos diferentes de lógica. Nesse sentido, Sinha (2016) afirma que a ambidestria tende a se tornar uma capacidade crítica no futuro, visto que pode permitir um desempenho superior da empresa tanto no curto quanto no longo prazo. Entretanto, o processo de construção e manutenção da capacidade ambidestra é um grande desafio para os gestores por abarcar elementos tidos como contraditórios pelos tradicionais processos organizacionais.

No campo da tecnologia, Vinekar, Slinkman e Nerur (2006) foram os autores que se apropriaram dos conceitos de ambidestria advindos do campo da estratégia e somaram aos aspectos levantados pela literatura a adoção simultânea de diferentes metodologias de desenvolvimento como característica de ambidestria em empresas de tecnologia. Segundo esses autores, a adoção de metodologias tradicionais de trabalho estaria relacionada à dimensão de exploração; portanto, a incorporação de metodologias ditas ágeis comporia a dimensão de explotação.

\subsection{Hipóteses}

De acordo com os estudos de Smith e Tushman (2005), a ambidestria é o caminho para o equilíbrio das práticas empresariais 
de maneira a atingir distinção de mercado, tendo impacto benéfico quando implementada e seguida por todos os níveis organizacionais. Ao assumir um comportamento ambidestro, a organização incorpora a possibilidade de sucesso e sobrevivência mercadológica, mas também suscita tensões e dificuldades de conciliação de ações e processos (ANDRIOPOULOS; LEWIS, 2009).

Nesse sentido, o constante processo de tomada de decisões, considerando a ambidestria empresarial, agrega riscos devido às incertezas ambientais próprias do mercado. E por ser a assunção de riscos ação inerente ao posicionamento empreendedor (LAZZAROTTI et al., 2015), compreender a relação entre esses construtos origina a primeira hipótese desta pesquisa: H1: A dimensão risco da orientação empreendedora correlaciona-se com a ambidestria.

O conceito de destruição criativa de Schumpeter (1997) destaca o constante movimento de criação e recriação a que as empresas estão sujeitas e dá significado à dimensão inovação da orientação empreendedora. Nesse contexto, a capacidade ambidestra se apresenta como uma constante troca entre os processos organizacionais de explotar capacidades existentes e explorar novas, ou seja, alcançar o equilíbrio entre o adequado para manter sua viabilidade atual e sua viabilidade futura (LEVINTHAL; MARCH, 1993). A inovação apresenta-se nesse processo e assim é proposta a segunda hipótese: H2: A dimensão inovação da orientação empreendedora correlaciona-se com a ambidestria.

O desenvolvimento de habilidades para identificar, implementar e renovar produtos, serviços e processos é inerente à busca por vantagens competitivas no longo prazo. Segundo Wei, Zhao e Zhang (2014), a ambidestria se apresenta como caminho possível para esse aumento da capacidade de adaptação da empresa em face das mudanças de mercado. Assim, reconhecer a necessidade constante de mudanças e antecipar-se a elas demonstra o grau de proatividade dos empreendedores que desejam estar a frente dos seus concorrentes (FAORO; ABREU, 2016). Baseando-se nesses aspectos, tem-se a terceira hipótese: H3: A dimensão proatividade da orientação empreendedora correlaciona-se com a ambidestria.

Miller e Friesen (1982) afirmam que o gestor empreendedor torna sua empresa diferente das demais. De acordo com Shane e 
Venkataraman (2000) o empreendedorismo está intimamente ligado à capacidade das organizações em analisar o mercado e enxergar as oportunidades existentes. De acordo com Purvee e Enkhtuvshin (2014), a habilidade dos gestores tomadores de decisão influencia a capacidade ambidestra da empresa em ambientes complexos. As informações e as trajetórias individuais de cada empreendedor criam esquemas mentais diferentes, determinando as tendências de escolha pela manutenção, pelo desenvolvimento ou pela adoção de ambas em relação a produtos e serviços. Com base nesse pensamento, a quarta hipótese é proposta: H4: A orientação empreendedora correlaciona-se com a ambidestria.

Conforme já levantado por March (1991), as ações de exploração estão ligadas à experimentação, à busca por novidades e à flexibilidade, com o objetivo de desenvolver novos produtos, serviços e processos. Nesse caminho criativo, a assunção de riscos é inerente às tomadas de decisão, seja pelo ineditismo da proposta, seja pelo acesso limitado a informações. Segundo os estudos de Hisrich, Peters e Shepherd (2014), a decisão de desenvolver um novo produto ou serviço se deve a ser embasada em informações suficientes que permitam avaliar a segurança da oportunidade, além de uma avaliação do grau de riscos que o empreendedor determina como aceitável.

Segundo He e Wong (2004), a dimensão exploração da ambidestria determina um comportamento organizacional baseado na busca, descoberta, tomada de decisão, experimentação e inovação constantes, por meio de novos produtos e processos. A criatividade e a inovação têm seus pilares na visão de oportunidades e antecipação das necessidades do mercado; assim, conforme afirmam Faoro e Abreu (2016), a inovação é um processo iterativo na natureza. Assim, propõem-se a quinta e sexta hipóteses: H5: A dimensão risco da orientação empreendedora correlaciona-se com a dimensão exploração da ambidestria; e H6: A dimensão inovação da orientação empreendedora correlaciona-se com a dimensão exploração da ambidestria.

Em uma perspectiva estratégica, Miller e Friesen (1982) afirmam que a dimensão proatividade tem a capacidade de modificar o ambiente por meio da inserção de novos produtos e serviços. Resgatando as afirmações de Wei, Zhao e Zhang (2014), a ambi- 
destria apresenta-se como um caminho possível para o processo de adaptação constante da empresa, e a exploração um processo de busca, de descoberta, de tomada de decisão, de experimentação e de inovação (HE; WONG, 2004). Nesse sentido, o processo de inovação pressupõe visão de oportunidades e sua implementação está relacionada à proatividade dos gestores na tomada de decisão que viabiliza o desenvolvimento constante (FAORO; ABREU, 2016). Dessa forma, formula-se a sétima hipótese: H7: A dimensão proatividade da orientação empreendedora correlaciona-se com a dimensão exploração da ambidestria.

De acordo com Tondolo et al. (2015), a orientação empreendedora tem se destacado no campo da estratégia como elemento impulsionador de inovação empresarial. Ao relacionar inovação, proatividade e assunção de riscos, temos caracterizado a orientação empreendedora caminhando ao encontro das afirmações de He e Wong (2004) relativas à dimensão exploração da ambidestria no que tange às atividades de busca por novas oportunidades e da tomada de decisão. Nesse sentido, propõe-se a oitava hipótese: H8: A orientação empreendedora correlaciona-se com a dimensão exploração da ambidestria.

Considerando-se a mudança constante do ambiente complexo no qual as empresas estão inseridas, mesmo as ações de aprimoramento, escolha, implementação e realização dos processos da organização estão sujeitas aos riscos da incerteza (TUSHMAN; O'REILLY, 1996). Assim, a decisão de assumir esses riscos sempre deve ser analisada conforme o rol de informações disponíveis e acessíveis ao tomador de decisões de maneira a permitir uma gestão dentro dos limites individuais e empresariais aceitáveis. Nesse mesmo sentido, a dimensão inovação permeia as ações de explotação da empresa, quando analisada sob a ótica de constante aprimoramento empresarial. Pressupõe-se relação entre a assunção dos riscos e inovação e as ações de explotação, haja vista a natureza dessa dimensão ambidestra. Assim, propõem-se a nona e a décima hipóteses: H9: A dimensão risco da orientação empreendedora correlaciona-se com a dimensão explotação da ambidestria; e H10: A dimensão inovação da orientação empreendedora correlaciona-se com a dimensão explotação da ambidestria. 
Segundo Lumpkin e Dess (1996), a dimensão proatividade tem como base a iniciativa do empreendedor em antecipar as necessidades e buscar novas oportunidades de mercado. De acordo com Faoro e Abreu (2016), a proatividade pode ser reconhecida conforme o nível de antecipação perante os concorrentes que o tomador de decisões está disposto a posicionar-se. Dessa forma, propõe-se a décima primeira hipótese: H11: A dimensão proatividade da orientação empreendedora correlaciona-se com a dimensão explotação da ambidestria.

Revisitando os escritos de Duncan (1974) verificam-se as características de implementação para criação da dimensão exploração da ambidestria, com um grau maior de formalização e menor complexidade em relação à exploração. Nesse contexto, a influência do comportamento do gestor sobre as ações ambidestras tende a definir as trajetórias empresariais adotadas (PURVEE; ENKHTUVSHIN, 2014). Ante o exposto, pressupõe-se correlação entre a orientação empreendedora e a dimensão explotação da ambidestria, compondo a décima segunda hipótese: H12: A orientação empreendedora correlaciona-se com a dimensão explotação da ambidestria.

Seguindo a estrutura proposta, na sequência serão abordados os procedimentos metodológicos utilizados para o desenvolvimento da pesquisa.

\section{Procedimentos Metodológicos}

O estudo foi elaborado com base na pesquisa quantitativa e técnica survey. A amostra, por conveniência, foi formada por 153 profissionais da área de Tecnologia da Informação dos estados do Rio Grande do Sul, São Paulo, Minas Gerais, Rio de Janeiro, Rondônia e outros não identificados. Os dados foram coletados e organizados por meio da ferramenta TypeForm para preenchimento online pelo público-alvo no período de julho a agosto de 2016.

Para a coleta dos dados relacionados à orientação empreendedora foram utilizados os questionários validados por Bolton e Lane (2012), composto por dez questões divididas nas dimensões: a) Risco, indicadores Ris1, Ris2 e Ris3; b) Inovação, indicadores Ino1, Ino2, Ino3 e Ino4; e c) Proatividade, indicadores Pro1, Pro2 e Pro3. Foi utilizada escala ordinal variando entre discordância total 
(1) até a plena concordância (7). O Quadro 1 traz os construtos, sua dimensão e os itens correspondentes.

Quadro 1: Itens construto orientação empreendedora individual

\begin{tabular}{|c|c|c|}
\hline Construto & Dimensão & Item \\
\hline \multirow{10}{*}{ 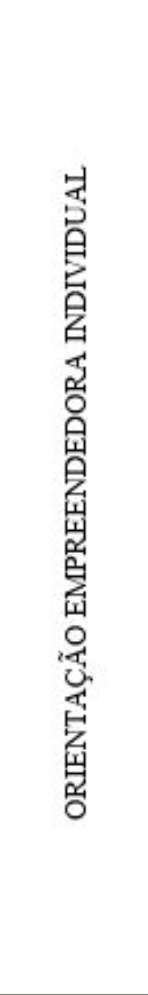 } & \multirow{3}{*}{ 递 } & $\begin{array}{l}\text { RIS1 - Eu gosto de realizar ações ousadas, me aventurando no } \\
\text { desconhecido. }\end{array}$ \\
\hline & & $\begin{array}{l}\text { RIS2 - Eu estou disposto a investir muito tempo e/ou dinheiro } \\
\text { em algo que pode gerar um alto retorno. }\end{array}$ \\
\hline & & $\begin{array}{l}\text { RIS3 - Eu tenho tendência a agir "corajosamente" em situações } \\
\text { em que o risco está envolvido. }\end{array}$ \\
\hline & \multirow{4}{*}{ 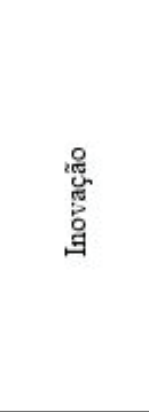 } & $\begin{array}{l}\text { INO1 - Eu gosto de tentar atividades novas e incomuns, mas que } \\
\text { não são necessariamente arriscadas. }\end{array}$ \\
\hline & & $\begin{array}{l}\text { INO2 - Em geral, eu prefiro atuar em projetos originais com } \\
\text { abordagens únicas em vez de projetos que utilizam abordagens já } \\
\text { utilizadas antes. }\end{array}$ \\
\hline & & $\begin{array}{l}\text { INO3 - Eu prefiro tentar a minha própria maneira de aprender } \\
\text { coisas novas em vez de fazê-lo como todo mundo faz. }\end{array}$ \\
\hline & & $\begin{array}{l}\text { INO4 - Eu sou a favor de abordagens experimentais e originais } \\
\text { de resolução de problemas em vez de usar métodos geralmente } \\
\text { usados pelos demais para resolver os seus problemas. }\end{array}$ \\
\hline & \multirow{3}{*}{ 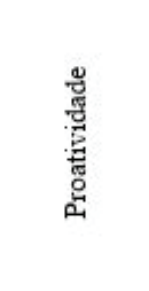 } & $\begin{array}{l}\text { PRO1 - Eu costumo agir em antecipação aos problemas futuros, } \\
\text { necessidades ou mudanças. }\end{array}$ \\
\hline & & PRO2 - Eu costumo planejar com antecedencia os projetos. \\
\hline & & $\begin{array}{l}\text { PRO3 - Eu prefiro "correr na frente" e colocar em andamento os } \\
\text { itens do projeto em vez de sentar e esperar por alguém para fazê- } \\
\text { lo. }\end{array}$ \\
\hline
\end{tabular}

Fonte: Adaptado de Bolton e Lane (2012, p. 229).

Para a coleta dos dados referentes à ambidestria organizacional foi utilizado o instrumento construído por Jansen (2005). O questionário foi aplicado em outras pesquisas como as de Silveira-Martins, Rossetto e Añaña (2013) e as de Silveira-Martins e Vaz (2016), demonstrando sua consistência e aplicabilidade.

O questionário é composto por 14 questões codificadas como Expr1 a Expr7 para as perguntas relacionadas à exploração; e Expt1 a Expt7 para explotação. Diferentemente da escala anterior, utilizou- 
se uma escala entre 1 (discordância total) e 6 (concordância total) às ações das fábricas de software, conforme modelo validado. $\mathrm{O}$ Quadro 2 mostra itens do construto ambidestria organizacional.

\section{Quadro 2: Itens construto ambidestria organizacional}

\begin{tabular}{|c|c|c|}
\hline Construto & Dimensão & Item \\
\hline \multirow{14}{*}{ 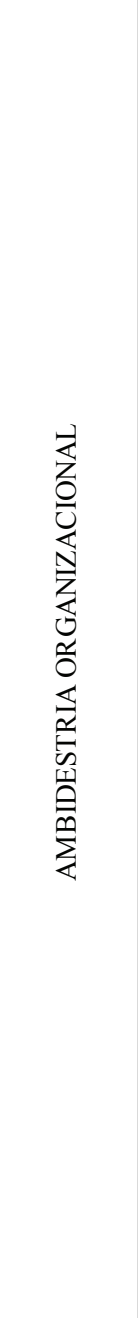 } & \multirow{7}{*}{ 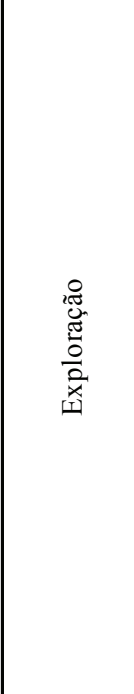 } & $\begin{array}{l}\text { EXPR1- A empresa aceita exigências que vão além de produtos e serviços } \\
\text { existentes. }\end{array}$ \\
\hline & & EXPR2 - A empresa cria novos produtos e serviços. \\
\hline & & $\begin{array}{l}\text { EXPR3 - A empresa experimenta novos produtos e serviços no mercado } \\
\text { local. }\end{array}$ \\
\hline & & $\begin{array}{l}\text { EXPR4 - A empresa comercializa produtos e serviços que são } \\
\text { completamente novos. }\end{array}$ \\
\hline & & $\begin{array}{l}\text { EXPR5 - A empresa frequentemente utiliza novas oportunidades em } \\
\text { novos mercados. }\end{array}$ \\
\hline & & $\begin{array}{l}\text { EXPR6 - Regularmente, a empresa utiliza novos clientes e novos } \\
\text { mercados. }\end{array}$ \\
\hline & & $\begin{array}{l}\text { EXPR7 - Regularmente, a empresa busca se aproximar de novos clientes } \\
\text { em novos mercados. }\end{array}$ \\
\hline & \multirow{7}{*}{ 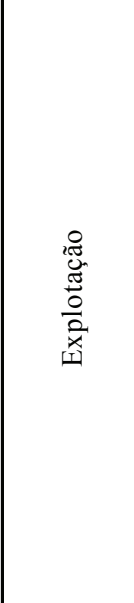 } & $\begin{array}{l}\text { EXPT1 - A empresa frequentemente melhora o fornecimento de produtos } \\
\text { e serviços existentes. }\end{array}$ \\
\hline & & $\begin{array}{l}\text { EXPT2 - A empresa regularmente implementa pequenas adaptações de } \\
\text { produtos e serviços existentes. }\end{array}$ \\
\hline & & $\begin{array}{l}\text { EXPT3 - A empresa introduz melhorias nos produtos e serviços existentes } \\
\text { no mercado local. }\end{array}$ \\
\hline & & $\begin{array}{l}\text { EXPT4 - A empresa melhora a eficiência da provisão de produtos e } \\
\text { serviços. }\end{array}$ \\
\hline & & $\begin{array}{l}\text { EXPT5 - A empresa aumenta a economia de escala em mercados } \\
\text { existentes. }\end{array}$ \\
\hline & & EXPT6 - A empresa amplia serviços para os clientes existentes. \\
\hline & & $\begin{array}{l}\text { EXPT7 - Para a empresa, a redução dos custos dos processos internos é } \\
\text { um objetivo importante. }\end{array}$ \\
\hline
\end{tabular}

Fonte: Adaptado de Jansen (2005, p. 95). 
Para análise dos dados, em um primeiro momento foi realizado teste de normalidade dos dados tabulados com base na medida de centralidade, de Kolmogorov-Smirnov com correção de significância de Lilliefors, conforme ensinamentos de Fávero et al. (2009). A existência ou falta de normalidade da distribuição dos dados permite definir o tipo de testes estatísticos a serem realizados. Na sequência, em função dos resultados do teste, optou-se pela realização da correlação de Spearman's. Tendo como base a metodologia apresentada, a seguir serão detalhados os resultados obtidos.

\section{ANÁlise dos DADOS}

Considerando a amostra de 153 questionários, antes de realizar a análise de correlação entre os construtos, foram realizados os testes de normalidade de Kolmogorov-Smirnov com os escores fatoriais gerados das dimensões da orientação empreendedora e da ambidestria. Nesse sentido, o teste de normalidade sinalizou distribuições não normais para todas as variáveis analisadas, conforme pode ser observado no Quadro 3.

Quadro 3: Teste de normalidade

\begin{tabular}{|l|c|c|c|}
\hline \multirow{2}{*}{\multicolumn{2}{|c|}{ VARIÁVEL }} & \multicolumn{3}{c|}{ KOLMOGOROV-SMIRNOV } \\
\cline { 2 - 4 } & Es tatís tica & GL & Valor-P \\
\hline Risco (RIS) & 0,101 & 153 & 0,001 \\
\hline Inovatividade (INO) & 0,135 & 153 & 0 \\
\hline Proatividade (PRO) & 0,156 & 153 & 0 \\
\hline Orientação Empreendedora (OE) & 0,086 & 153 & 0,008 \\
\hline Ambidestria (AMB) & 0,096 & 153 & 0,001 \\
\hline Exploração (EXPR) & 0,092 & 153 & 0,003 \\
\hline Explotação (EXPT) & 0,108 & 153 & 0 \\
\hline
\end{tabular}

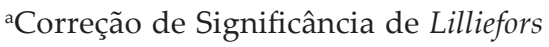

Fonte: Dados da pesquisa.

Para que as distribuições de dados analisados fossem consideradas simétricas (normais), os valores parâmetros (valor-P) deveriam apresentar uma significância estatística superior a 0,05, o que não ocorreu, conforme já apresentado no Quadro 3. 
Diante da ausência de normalidade, optou-se pela realização de teste não paramétrico para verificar a existência de correlação entre os construtos. O teste de correlação Spearman foi utilizado para determinar as relações entre as variáveis. A associação entre as variáveis é analisada de acordo com o coeficiente de correção ( $Q$ (rho)), a qual varia entre -1 e 1 , sendo o sinal a direção, e o valor a magnitude da correlação - quanto mais próximo do centro (zero), menor o grau de associação entre as variáveis. Coeficientes positivos indicam que quando uma variável aumenta a outra também se eleva, e coeficientes negativos indicam associação inversa, ou seja, quando uma variável aumenta a outra diminui (FIGUEIREDO et al., 2014).

Utilizando-se das escalas estabelecidas por Barbetta (2012), identificou-se que todas as correlações encontradas foram fracas, ou seja, possuem valor de @ (rho) abaixo de 0,30. A orientação empreendedora possui coeficiente de correlação positiva com a ambidestria apresentando $\varrho=0,155$ e não significância estatística $(\alpha=0,05<p=0,056)$. Nessa mesma linha a correlação da orientação empreendedora com a dimensão explotação da ambidestria também se apresentou positiva $(\varrho=0,069)$ e não significante com $\alpha=0,05<p=0,339$. A correlação entre as variáveis orientação empreendedora e a dimensão exploração da ambidestria se mostrou positiva com $\varrho=0,205$, mas possui significância estatística com $\alpha$ $=0,05>\mathrm{p}=0,011$.

Com relação à dimensão assunção de riscos, apresentou correlação positiva e não significante $(p>0,05)$ em relação a todas variáveis testadas. Já a inovação apresentou correlação positiva de $\mathrm{Q}=0,254$ com a ambidestria com significância a $\alpha=0,05>\mathrm{p}=0,002$, assim como correlação positiva ( $\varrho=0,275)$ e significante $(\alpha=0,05>$ $\mathrm{p}=0,001)$ com a dimensões exploração. Os resultados de correlação obtidos se exibem no Quadro 4. 
Quadro 4: Correlação de Spearman's

\begin{tabular}{|c|c|c|c|c|}
\hline HIPÓTESE & ASSOCIAÇÃO & $\rho$ (rho) & VALOR-P & $\mathbf{N}$ \\
\hline H1 & RIS $\Leftrightarrow$ AMB & $-0,013$ & 0,871 & \multirow{12}{*}{15} \\
\hline $\mathrm{H} 2$ & $\mathrm{INO} \Leftrightarrow \mathrm{AMB}$ & 0,254 & 0,002 & \\
\hline $\mathrm{H} 3$ & $\mathrm{PRO} \Leftrightarrow \mathrm{AMB}$ & 0,226 & 0,005 & \\
\hline $\mathrm{H} 4$ & $\mathrm{OE} \Leftrightarrow \mathrm{AMB}$ & 0,155 & 0,056 & \\
\hline H5 & RIS $\Leftrightarrow$ EXPR & 0,043 & 0,594 & \\
\hline H6 & INO $\Leftrightarrow$ EXPR & 0,275 & 0,001 & \\
\hline $\mathrm{H} 7$ & PRO $\Leftrightarrow$ EXPR & 0,281 & 0 & \\
\hline $\mathrm{H} 8$ & $\mathrm{OE} \Leftrightarrow \mathrm{EXPR}$ & 0,205 & 0,011 & \\
\hline H9 & RIS $\Leftrightarrow$ EXPT & $-0,093$ & 0,254 & \\
\hline H10 & INO $\Leftrightarrow$ EXPT & 0,18 & 0,026 & \\
\hline H11 & $\mathrm{PRO} \Leftrightarrow \mathrm{EXPT}$ & 0,147 & 0,069 & \\
\hline $\mathrm{H} 12$ & $\mathrm{OE} \Leftrightarrow \mathrm{EXPT}$ & 0,069 & 0,399 & \\
\hline
\end{tabular}

Fonte: Dados da pesquisa.

Diante dos resultados identifica-se que as hipóteses $\mathbf{H 2}$, $\mathrm{H6}$ e H10, que tratam da dimensão inovação da orientação empreendedora, foram suportadas, com associação positiva fraca com a ambidestria e suas dimensões. Esse resultado corrobora os estudos de Levinthal e March (1993), He e Wong (2004) e Faoro e Abreu (2016) sobre o comportamento empresarial ambidestro ser uma constante busca por descobertas e inovações mediante novos produtos, serviços e processos, e pela busca por equilíbrio entre a sua viabilidade atual e futura no mercado. A inovação passa a ser uma das bases de manutenção e renovação das empresas por meio da exploração e explotação de suas habilidades e potencialidades de mercado.

As hipóteses H1, H5 e H9, que afirmam a relação entre a dimensão assunção de risco com a ambidestria e suas dimensões, não puderam ser confirmadas pois não apresentaram significância estatística. A fraca correlação negativa encontrada em relação à ambidestria (H1) e a dimensão explotação (H9) demonstra predisposição de diminuição da assunção de riscos quando há um aumento da variável correlacionada. Esses resultados vão de encontro à afirmação de Lazzarotti et al. (2015) de que assumir riscos é inerente ao empreendedor e à tomada de decisões. Vale ressaltar que em relação 
à exploração (H5) a correlação foi positivamente fraca, demonstrando uma tendência de se assumirem riscos durante o desenvolvimento de um novo produto, processo ou serviço. Entretanto, devido à não confirmação estatística, os resultados não podem ser confirmados.

Considerando as pesquisas de Faoro e Abreu (2016), as correlações positivas encontradas nas afirmações das hipóteses H3 e H7 corroboram as afirmações de que a necessidade de constante mudança e antecipação de cenários está ligada ao grau de proatividade assumido pelo gestor empreendedor. A ambidestria apresenta-se como um caminho no aumento da capacidade de adaptação empresarial ante as incertezas mercadológicas (WEI; ZHAO; ZHANG, 2014). No tocante à hipótese H11, cuja afirmativa é de que existe relação entre a dimensão proatividade da orientação empreendedora e a dimensão explotação da ambidestria, esta não foi confirmada

Quando analisada a hipótese $\mathbf{H} 4$ sobre a correlação entre orientação empreendedora e ambidestria, não foi possível confirmar a afirmação nesta pesquisa. Nesse mesmo sentido, a hipótese $\mathbf{H 8}$ não confirmou a relação entre a orientação empreendedora e a dimensão explotação da ambidestria. Esses resultados vão de encontro à afirmação de Purvee e Enkhtuvshin (2014) sobre a influência dos gestores tomadores de decisão na capacidade ambidestra da empresa. Percebe-se que a implementação criativa da explotação não seria dependente de ações empreendedoras dos gestores. Já a hipótese H12, que afirma correlação entre a orientação empreendedora e a dimensão exploração, foi suportada,

O Quadro 5 resume os resultados relativos às hipóteses testadas.

Considerando as hipóteses que foram possíveis suportar, destaca-se a correlação positiva da dimensão inovação da orientação empreendedora com a ambidestria e suas dimensões, por apresentar significância estatística em todas as hipóteses testadas. Esse resultado parece coerente com a natureza tecnológica dos profissionais que fizeram parte da amostra pesquisada.

Vale ressaltar que, de acordo com Lazzarotti et al. (2015), a natureza e o contexto no qual os componentes da orientação empreendedora se encontram influenciam a configuração das dimensões, apresentando maior ou menor aderência a outros construtos estratégicos. Assim, o caráter tecnológico e inovador do objeto da 
pesquisa tende a reunir características com algumas dimensões em detrimento de outras.

Quadro 5: Resultado analise das hipóteses

\begin{tabular}{|c|c|c|}
\hline HIPÓTESE & ASSOCIAÇÃo & Resultado \\
\hline $\mathrm{H} 1$ & RIS $\Leftrightarrow$ AMB & Não suportada \\
\hline $\mathrm{H} 2$ & $\mathrm{INO} \Leftrightarrow \mathrm{AMB}$ & Suportada \\
\hline $\mathrm{H} 3$ & $\mathrm{PRO} \Leftrightarrow \mathrm{AMB}$ & Suportada \\
\hline $\mathrm{H} 4$ & $\mathrm{OE} \Leftrightarrow \mathrm{AMB}$ & Não suportada \\
\hline $\mathrm{H} 5$ & $\mathrm{RIS} \Leftrightarrow \mathrm{EXPR}$ & Não suportada \\
\hline $\mathrm{H} 6$ & $\mathrm{INO} \Leftrightarrow \mathrm{EXPR}$ & Suportada \\
\hline $\mathrm{H} 7$ & $\mathrm{PRO} \Leftrightarrow \mathrm{EXPR}$ & Suportada \\
\hline $\mathrm{H} 8$ & $\mathrm{OE} \Leftrightarrow \mathrm{EXPR}$ & Suportada \\
\hline $\mathrm{H} 9$ & $\mathrm{RIS} \Leftrightarrow \mathrm{EXPT}$ & Não suportada \\
\hline $\mathrm{H} 10$ & $\mathrm{INO} \Leftrightarrow \mathrm{EXPT}$ & Suportada \\
\hline $\mathrm{H} 11$ & $\mathrm{PRO} \Leftrightarrow \mathrm{EXPT}$ & Não suportada \\
\hline $\mathrm{H} 12$ & $\mathrm{OE} \Leftrightarrow \mathrm{EXPT}$ & Não suportada \\
\hline
\end{tabular}

Fonte: Dados da pesquisa.

\section{CONSIDERAÇõES FINAIS}

A compreensão da predisposição dos indivíduos para a atividade empresarial em conjunto com as características ambidestras organizacionais tem se apresentado extremamente relevante no atual cenário econômico e no contexto competitivo de mercado. Nesse sentido, a orientação empreendedora torna-se um forte caminho estratégico quando avaliada em nível individual e em conjunto com as ações ambidestras organizacionais.

Visando a contribuir com os profissionais de tecnologia na tomada de decisões relativas às parcerias de negócios, à definição de metodologias de trabalho e a outras ações estratégicas do cotidiano, a presente pesquisa teve por objetivo verificar a relação entre as dimensões da orientação empreendedora individual e as dimensões da ambidestria organizacional. 
Vale ressaltar que a pesquisa se limita na amostra investigada, a qual, embora consistente para as análises propostas, está restrita a um público específico e restrito, razão por que os resultados não podem ser generalizados para um contexto diferente. Assim, sugere-se a ampliação da pesquisa em outros segmentos profissionais de maneira a diversificar as áreas e verificar se os resultados encontrados se mantêm ou se haverá alteração conforme contexto mercadológico. A realização de comparações interculturais poderia agregar informações de extrema relevância, dadas as disparidades culturais entre territórios em relação a atitudes de risco, políticas governamentais ou formação histórica da sociedade e sua relação com a orientação empreendedora e a ambidestria organizacional.

\section{REFERÊNCIAS}

ANDRIOPOULOS, C.; LEWIS, M. W. Exploitation-Exploration Tensions and Organizational Ambidexterity: Managing paradoxes of innovation. Organization Science, v. 20, n. 4, p. 696-717, 2009.

BARBETTA, P. A. Estatística aplicada às Ciências Sociais. 8. ed. rev. Florianópolis: Ed. da UFSC, 2012.

BLEDOW, R.; FRESE, M.; ANDERSON, N.; EREZ, M.; FARR, J. A Dialectic Perspective on Innovation: Conflicting Demands, Multiple Pathways, and Ambidexterity. Industrial and Organizational Psychology, v. 2, n.3, p. 305-337, 2009.

BOLTON, D. L.; LANE, M. D. Individual entrepreneurial orientation measurement instrument. Education + Training, v. 54, n. 2/3, p. 219-233, 2012.

CARVALHO JR, A. M. A Política Industrial e o BNDES. Revista do BNDES, v. 12, n. 23, p. 17-28, jun. 2005.

COVIN, J. G.; SLEVIN, D. P. A conceptual model of entrepreneurship as firm behavior. Entrepreneurship: Theory \& Practice, United States, v. 16, n. 1, p. 7-25, Fall 1991.

DICKSON, P. H.; WEAVER, K. M. The role of the institutional environment in determining firm orientations towards entrepreneurial behavior. International Entrepreneurship Management Journal, v. 4, n. 4, p. 467-483, 2008.

DUNCAN, R.B. The ambidextrous organization: designing dual structures for innovation. Un: KILMAN, R.H.; PONDY, L.R.; SLEVIN, D.P. The management of organization design: strategies and implementation. v.1, n.1, New York: University of Pittsburgh, p.167-188, 1974.

FAORO, R.; ABREU, M. A Orientação para aprendizagem e orientação ao empreendedorismo na vantagem do produto. Revista de Administração da UNIFATEA, v. 12, n.12, p. 23-39, 2016.

FÁVERO, L. P. L.; BELFIORE, P. P.; CHAN, B. L.; SILVA, F. L. Análise de Dados: Modelagem Multivariada para Tomada de Decisões. Rio de Janeiro: Elsevier, 2009. 
FERREIRA, F. A. F.; JALALI, M. S.; BENTO, P.; FERREIRA, J. J. M. Enhancing individual entrepreneurial orientation measurement using a metacognitive decision making-based framework. International Entrepreneurship and Management Journal, v. 12, n. 1, p. 1-20, 2016.

FIGUEIREDO FILHO, D. B.; ROCHA, E. C.; SILVA JR, J.; PARANHOS, R.; NEVES, J. A. B.; SILVA, M. B. Desvendando os mistérios do coeficiente de correlação de Pearson. Leviathan Cadernos de Pesquisa Política, n. 8, p. 66-95, 2014.

FRANKLIN, M. A. Estrutura organizacional, orientação para exploitation e exploration e tipos de inovação em institutos de pesquisa de desenvolvimento tecnológico. $217 \mathrm{f}$. Tese. (Doutorado em Administração) - Programa de Pós-Graduação em Administração de Empresas, Universidade Presbiteriana Mackenzie, São Paulo, 2010.

FREITAS, H.; MARTENS, C. D. P.; BOISSIN, J. P.; BEHR, A. Elementos para guiar ações visando à orientação empreendedora em organizações de software. Rev. Adm. São Paulo, v. 47, n. 2, p. 163-179, jun. 2012.

GOKTAN, A. B.; GUPTA, V. K. Sex, gender, and individual entrepreneurial orientation: evidence from four countries. International Entrepreneurship and Management Journal, v. 9, n.1, p. 1-18, 2013.

GUPTA, A. K.; SMITH, K. G.; SHALLEY, C. E. The interplay between exploration and exploitation. Academy of Management Journal, v. 49, n.4, p. 693-708, 2006.

HAN, M.; CELLY, N. Strategic ambidexterity and performance in international new ventures. Canadian Journal of Administrative Science, Chichester, v. 25, n. 4, p. 335-349, dec. 2008.

HE, Z-L.; WONG, P-K. Exploration vs. exploitation:an empirical test of the ambidexterity hypothesis. Organization Schience, v.14, n.4, p. 484-494, 2004.

HISRICH, R. D.; PETERS, M. P.; SHEPHERD, D. A. Empreendedorismo. Tradução de Francisco Araújo da Costa. Porto Alegre: AMGH Editora Ltda, 2014.

HSU, C.-W.; LIEN, Y.-C.; CHEN, H. International ambidexterity and firm performance in small emerging economies. Journal of Word Business, v. 48, n. 1, 58-67, jan. 2013.

JANSEN, J. J. P. Ambidextrous organizations: a multiple-level study of absorptive capacity, exploratory and explotative innovation and performance. 196 f. PHD Thesis - Erasmus Research Institute of Management, Erasmus University Rotterdam, Rotterdam, 2005.

KOLLMANN, T.; CHISTOFOR, J.; KUCKERTZ, A. Explaining individual entrepreneurial orientation: conceptualization of a cross-cultural framework. International Journal of Entrepreneurship and Small Business, v. 4, n.3, p. 325-340, 2007.

LAZZAROTTI, F.; SILVEIRA, A. L. T.; CARVALHO, C. E.; ROSSETTO, C. R.; SYCHOSKI, J. C. Orientação Empreendedora: Um estudo das dimensões e sua relação com desempenho em empresas graduadas. RAC, Rio de Janeiro, v. 19, n. 6, p. 673-695, nov./dez. 2015.

LIN, Z.; YANG, H.; DEMIRKAN, I. The performance consequences of ambidexterity in strategic alliance formations: empirical investigation and computational theorizing. Management Science, Linthicum, v. 10, n. 5, p. 1645-1658, oct. 2007. 
LUMPKIN, G. T.; DESS, G. G. Clarifying the entrepreneurial orientation construct and linking it to performance. Academic of Management Review, United States, v. 21, n. 1, p. 135-172, jan. 1996.

MARCH, J. G. Exploration and exploitation in organization learning. Organization Science, Linthicum, v. 2, n. 1, p. 71-87, feb. 1991.

MILLER, D. The correlates of entrepreneurship in three types of firms. Manage. Sci. v.29, n.7, p. 770-791, 1983.

MILLER, D.; FRIESEN, P. H. Innovation in conservative and entrepreneurial firms: two models of strategic momentum. Strategic Management Journal, v. 3, n. 1, p. 1-26, 1982.

MORRIS, M. H. Entrepreneurial Intensity: Sustainable Advantages for Individuals, Organizations and Societies. Westport, CT, USA: Greenwood Publishing Group, Incorporated, 1998.

NUZZO, R. Scientific method: Statistical error. Nature, v. 506, n. 7487, p. 150-152, feb. 2014.

PARMENTIER, G.; PICQ, T. Managing creative teams in small ambidextrous organizations: The case of videogames. International Journal of Arts Management. v. 16, n. 1, p. 16-30, 2016.

PURVEE, A.; ENKHTUVSHIN, D. Transformational Leadership and Managers Ambidexterity: Mediating Role of Environmental Dynamism. International Journal of Innovation, Management and Technology, v. 5, n. 6, p. 434-437, 2014.

SCHUMPETER, J. A. Teoria do desenvolvimento econômico. Tradução de Maria Sílvia Possas. São Paulo: Nova Cultural, 1997.

SHANE, S.; VENKATARAMAN, S. The Promisse of Emtrepreneurship as Field of Resarch. The Academy of Management Review, v. 25, n. 1, p. 217-227, 2000.

SILVEIRA-MARTINS, E.; ROSSETTO, C. R.; AÑAÑA, E. Ambidestria, Exploração ou Explotação e seus efeitos no desempenho organizacional de vinícolas brasileiras. In: ENCONTRO DE ESTUDOS EM ESTRATÉGIA, 6., 2013, Rio Grande do Sul. Anais eletrônicos... Bento Gonçalves. ANPAD, 2013. Disponível em: <http://www.anpad.org.br/diversos/trabalhos/3Es/3es_2013/2013_3Es144.pdf>. Acesso em: 10 ago. 2016.

SILVEIRA-MARTINS, E.; VAZ, C. S. Orientação empreendedora e sua associação com as capacidades dinâmicas: um estudo em agroindústrias. Revista em Agronegócio e Meio Ambiente, Maringá, v. 9, n. 3, p. 509-529, jul./set. 2016.

SINHA, S. Managing an ambidextrous organization: balancing innovation and efficiency. Strategic Direction, v. 32, n.10, p. 35-37, 2016.

SMITH, W.K; TUSHMAN, M.L; Managing strategic contradictions: a top management model for managing innovation streams. Organization Science, v. 16, n.5, p. 522-236, 2005.

STEVENSON, H. H.; JARILLO, J. C. A paradigm of entrepreneurship: entrepreneurial management. Strategic Management Journal, Chichester, v. 11, n. 5, p. 17-27, sep. 1990.

TAATILA, V.; DOWN, S. Measuring entrepreneurial orientation of university students. Education Training, v. 54, n. 8/9, p. 744-760, 2012. 
TAYLOR, A.; HELFAT, C. E. Organizational linkages for surviving technological change: complementary assets, middle management, and ambidexterity. Organization Science, Linthicum, v. 20, n. 4, p. 718-739, jun. 2009.

TEECE, D. J. Explicating dynamic capabilities: the nature and microfoundations of (sustainable) enterprise performance, Strategic Management Journal, Malden, v. 28, n. 13, p. 1319-1350, aug. 2007.

TONDOLO, V. A. G.; TONDOLO, R. R. P.; CAMARGO, M. E.; BITENCOURT, C. C. Orientação empreendedora e capacidades dinâmicas em ambiente de inovação. Revista GEINTEC, v. 5, n. 3, p. 2452-2455, 2015

TUSHMAN, M. L.; O'REILLY, C. A. Ambidextrous Organizations: Managing evolutionary and revolutionary Change, California Management Review, v. 38, n.4, p. 8-30, 1996.

VINEKAR, V.; SLINKMAN, C.W.; NERUR, S. Can agile and traditional systems development approaches coexist? An ambidextrous view. Information Systems Management Journal, v. 23, n. 3, p. 31-42, 2006.

WEI, Z.; ZHAO, J.; ZHANG, C. Organizational ambidextery, market orientation, and firm performace. Journal of Engineering and Technology Management, v. 33, n.1, p. 134-153, 2014.

Recebido em: 19-12-2016

Aprovado em: 24-04-2017

Avaliado pelo sistema double blind review.

Editor: Elmo Tambosi Filho

Disponível em http://mjs.metodista.br/index.php/roc 